\title{
WOLF-RAYET POPULATIONS IN DWARF GALAXIES
}

\author{
LINDSEY F. SMITH \\ Mount Stromlo and Siding Spring Observatories \\ Australian National University \\ GPO Box 4, Canberra ACT 2601 \\ Australia
}

\begin{abstract}
The Wolf-Rayet (WR) feature at $4650 \mathrm{~A}$ is observed in about $10 \%$ of the dwarf galaxies with high surface brightness knots. The intensity of the feature implies the presence of tens to thousands of WR stars. Hbeta fluxes imply correspondingly large numbers of $O$ stars. The easily observed intensity ratio WR bump/Hbeta is a measure of the WR/O star numbers.
\end{abstract}

The metallicity of dwarf galaxies ranges from $\mathrm{Z}=\mathrm{Zo} / 30$ to $\mathrm{Zo} / 2$, or $\mathrm{O} / \mathrm{H}^{\prime \prime}=\log (\mathrm{O} / \mathrm{H})+12=7.4$ to 8.6. WR bump/Hbeta correlates with $\mathrm{O} / \mathrm{H}^{\prime \prime}$ and $\mathrm{O} / \mathrm{H}^{\prime \prime}>7.9$ appears to be a necessary condition for the presence of the WR feature. Giant HII regions in ordinary galaxies extend to higher than solar metallicities and, in extreme cases, $W R / O \approx 1$ are implied.

The subtypes present in giant HII regions in nearby galaxies appear to be exclusively late type WN and, occasionally, early type WC. Spectra of most BCD galaxies are compatible with a similar population. However, some high metallicity giant HII regions in large galaxies appear to have stronger NIII4640 relative to HeII4686 than occurs in WN subtypes in the Galaxy and the Magellanic Clouds.

The data needed for more detailed analysis of dwarf galaxy observations is collected.

\section{The Observations}

Dwarf galaxies are low mass, low luminosity systems; the dividing line from ordinary luminous galaxies is at about $M_{B}=-19$. Among the dwarfs, there is a subset with high surface brightness knots, having the appearance of giant HII regions. They were, in fact, first called extragalactic HII regions (Sargent and Searle 1970), but were subsequently found to be embedded in otherwise low surface brightness galaxies. They now go by the name of Blue Compact Dwarf (BCD) galaxies.

The explanation of these galaxies, which appears to be generally accepted, was given by Searle et.al. (1973) and by Gerola et.al. (1980); the BCD galaxies represent a phase of intense star formation in dwarf irregular (Im) galaxies. The regions of star formation appear to involve 1E3 to 1E5 ionising stars; i.e. from numbers a little larger to several orders of magnitude larger than for 30 Doradus. Furthermore, similar compact objects appear to exist up to $\mathbf{M}_{B} \approx-23$ involving 1E7 ionising stars (Hazard 1985). Such objects are very numerous, about 1 per square degree (Hazard 1985). Noting that 30 Dor is larger than any giant HII region in our own Galaxy, the rate of star formation in these regions is prodigious.

Giant and supergiant HII regions in the larger galaxies present observational similarities to those in $\mathrm{BCD}$ galaxies - they have unresolved cores containing large numbers of massive stars plus, of course, 
surrounding nebulosity. For this reason, they are often included in the same studies and will, accordingly, be included in the present review.

Dwarf galaxies are generally of low metallicity; ranging from $\mathrm{Zo} / 30(\mathrm{Zw} 18)$ to $\mathrm{Zo} / 2$; they show no large scale structure and no abundance gradients (Kunth 1985). The regions are small (or the order of $100 \mathrm{pc}$ ) so it is fair to assume that they are, or were, chemically homogeneous before the present burst of star formation commenced. From this derives one of the principle reasons for interest in the BCD galaxies they present an opportunity to study star formation at well defined and low $\mathrm{Z}$, with a very large number of stars being formed in the one burst. By the same token, giant HII regions in ordinary galaxies represent the same phenomenon at higher metallicities - another reason for their inclusion in the papers reviewed here.

Allen et.al. (1976) made the first observation of WR features in a dwarf galaxy, He2-10. The most prominent emission feature, characteristic of all subtypes of WR star, is the broad emission in the vicinity of $4650 \mathrm{~A}$. The flux contributed by one WR star to this feature is of the order of $1 \mathrm{E} 36 \mathrm{ergs} / \mathrm{sec}$ (Kunth and Sargent 1981; Arnault et.al. 1989, henceforth AKS). Figure 5 of Kunth and Schild (1986) shows the fluxes observed in the spectra of BCD galaxies and giant HII regions; they range from 1E38 to 1E41 ergs/sec, i.e. 100 to 100,000 WR stars ! It is plotted against $\mathrm{O} / \mathrm{H}^{n}$ (used as a shorthand here for $(\log \mathrm{O} / \mathrm{H}+12)$ and includes some high $\mathrm{Z}$ giant $\mathrm{HII}$ regions. The meaning of the (fairly good) correlation is ambiguous. The authors consider that a selection effect may be operating.

Metallicity, however, does appear to play a significant role, as shown by AKS. Their Figure 8, shows the ratio of the WR feature (called WRbump) to Hbeta versus $\mathrm{O} / \mathrm{H}^{\prime \prime}$. The Hbeta flux is a measure of the number of ionising photons and hence the total number of $O$ (and WR) stars. The upper limit of WR bump/Hbeta increases with $\mathrm{O} / \mathrm{H}^{\prime \prime}$, implying that high $\mathrm{O} / \mathrm{H}$ " is a necessary condition for high WR/O star numbers. It is also notable that the WR feature is only observed for $\mathrm{O} / \mathrm{H}^{\prime \prime}>$ 7.9. To first approximation, WR/O (numbers) $\approx \mathrm{WRbump/Hbeta} \mathrm{(intensity).} \mathrm{Note} \mathrm{that} \mathrm{the} \mathrm{values} \mathrm{of} \mathrm{the} \mathrm{latter} \mathrm{imply}$ $\mathrm{WR} / \mathrm{O} \approx 1$ for some of the high $\mathrm{O} / \mathrm{H}$ " regions in ordinary galaxies (eg. NGC5128 \#13, O/H" $\approx 9$, see Rosa and d'Odorico 1986 for population simulation; Mrk309 and NGC6764, see Osterbrock and Cohen 1982).

High $\mathrm{O} / \mathrm{H}$ " is not, however, a sufficient condition for a large WR presence; low values of WRbump/Hbeta also occur. An observational effect contributes - the nebulosity is more extended than the ionising cluster and (depending on resolution and slit size) can get excluded from the observation. However, the scatter is undoubtedly real and is expected (see below) since the WR lifetime is short compared to the $\mathrm{O}$ star lifetimes allowing the presence of $\mathrm{O}$ stars without any WR stars.

In giant HII regions in nearby galaxies, the subtypes which occur appear to be predominately WN6-7 and, less commonly, WN8 and WC4 (see D'Odorico et.al. 1983; Rosa and D'Odorico 1986; for 30 Dor see Walborn 1986; Melnick 1985a, Moffat et.al. 1987). A conspicuous contribution from WC spectra is unusual in dwarf galaxies (see eg. Kunth and Schild 1986). The latter authors stress that [FeIII] 4658 occurs frequently and, at low resolution, can masquerade as CIV 4650 creating a false impression of a WC contribution. In lower metallicity galaxies, the [FeIII] line is not conspicuous in published spectra - as would be expected. He2-10 is the clearest case of a dwarf galaxy where WC stars appear to contribute (but see the discussion in Section 2.4 below). However, in giant HII regions in "ordinary" galaxies there are many convincing examples of WC contribution (see eg. D'Odorico et.al. 1983). Rosa and D'Odorico (1986) note a strong correlation between regions where there has been a type II supernova and the presence of WC emission.

Subtypes of WR stars in the dwarf galaxies are mostly compatible with the presence of these subclasses. However, it will be shown below that spectra of some giant $\mathrm{HII}$ regions defy simulation with a simple combination of WN6-8 and WC4. 


\section{The expectations.}

I now propose to compare these observations with what we expect to see, based on stellar evolution calculations and observations of individual WR stars in nearby galaxies. Specifically:

* the number ratios of WR/OB and WC/WN as functions of metallicity (Section 2.1);

* the subtype distributions (Section 2.2);

* the calibration of the flux in the WR feature at 4650 A (Section 2.3);

* separation of WN and WC contributions using the CIV 5808 feature (Section 2.4);

* the question of whether WR stars are the same in all galaxies (Section 2.5).

\subsection{NUMBERS OF WOLF-RAYET STARS}

The numbers of WR stars relative to $O$ stars (from which they evolve) and the numbers of WC stars relative to the WN stars vary dramatically from one galaxy to another and within large galaxies as a function of radius. The cause of the variation has been attributed to metallicity (Smith 1968b, Maeder et.al. 1980) and to variations of the IMF (Conti et.al. 1983). The theoretical connection to metallicity is that higher mass loss rates for the progenitor $O$ star are predicted when the metallicity is high (Abbott 1982). This causes the star to reach the WR stage earlier in its evolution and thus increases the overall WR lifetime and the WC fraction of that lifetime (Maeder et.al. 1980; Maeder 1981).

The theoretical connection to the IMF is that only massive stars become (Population I) WR stars and so an increase in the relative numbers of massive stars will increase the WR/OB ratio. Both factors will have an effect. However, the evidence favouring metallicity as the dominant factor is now overwhelming. In short:

* The correlation of both WR/OB and WC/WN with metallicity is excellent (Smith 1988; AKS; Maeder 1990b). The criticism of the correlation (eg. Massey 1986; Massey and Conti 1983; Armandroff and Massey 1985) is that regions with similar O/H" to the LMC and SMC have different populations. The answer is (Smith and Maeder 1990) that, in the large spirals, metallicity at a given galactocentric radius covers a wide range (eg. Talent and Dufour 1979). Because higher $Z$ regions have more WR stars as well as more WC stars, the average WR population will be weighted towards values characteristic of a higher $Z$ value.

* The agreement between predicted and observed number ratios at different metallicity is excellent (Maeder $1990 \mathrm{~b}$ and this volume);

* The detailed explanation of the WC subtype distribution in terms of metallicity (Smith and Maeder 1990 , and see below) clinches the argument.

* I would further point out that regions of low $\mathrm{Z}$ are always low in WR numbers and the effect of $\mathrm{Z}$ on the IMF is generally suggested to favour high mass stars (eg. Scalo 1986; Terlevich 1985).

Thus the correlation found for dwarf galaxies and giant HII regions between the upper limit of WR bump/Hbeta and $\mathrm{O} / \mathrm{H}^{\text {" }}$ is appropriately interpreted by the AKS as due to more WR stars at higher metallicity. The $\mathrm{O} / \mathrm{H}^{\mathrm{n}}$ lower limit of 7.9 corresponds to $\mathrm{Z} \approx .002$ at which the models (Maeder 1990a,b) predict that only stars more massive that $85 \mathrm{Mo}$ attain WR status.

The large range of WRbump/Hbeta values at a given $\mathrm{O} / \mathrm{H}^{n}$ is qualitatively explained by the evolution of a star burst region. AKS calculate WR/O and WRbump/Hbeta as a function of time. For continuous star formation, the numbers are, of course, approximately constant. However, for the burst scenario, there is a WR phase which commences at an age of $\sim 3 \mathrm{E} 6 \mathrm{yrs}$, when the most massive stars become WR stars, and ends with the demise of the lowest mass stars which are capable of loosing their $\mathrm{H}$ envelopes in their lifetime; both times are $\mathrm{Z}$ dependent, the end point more than the starting point. 
At low $\mathrm{Z}$, the cluster's WR phase is very short, $\sim 6 \mathrm{E} 5$ yrs at $\mathrm{ZO} / 10$, because only the most massive stars attain WR status. The lifetime of the lowest mass $\mathrm{O}$ stars is $\sim 6 \mathrm{E} 6 \mathrm{yrs}, 10$ times longer; this satisfactorily accounts for the observation that only $\sim 1 / 10$ of the BCD galaxies have a detected WR feature.

For solar Z, the WR phase lasts 3E6 yrs; during that time the ratio of WR/O star numbers and WRbump/Hbeta increase, as the number of WR stars decreases more slowly than the number of remaining $O$ stars. The WR population decreases more slowly because, as lower mass stars enter the WR phase, their shorter WR lifetime is offset by their larger numbers.

30 Dor is noteworthy in this regard. Numbers assembled by AKS indicate that the 30 Dor region has a WR/O ratio approximately equal to the average for the whole LMC. However, the age is estimated at only 2E6 yrs (Melnick 1985b), less than expected for the onset of the WR phase, even for a 120 Mo star. The WR population in 30 Dor may increase dramatically in the next million years.

AKS's population simulations do not generate WRbump/Hbeta values as high as observed. This is remedied, in part, by higher mass loss rates used in more recent evolutionary models (Maeder 1990a,b). AKS demonstrate that the "burst scenario" generates WR/O values an order of magnitude higher than continuous formation. It remains to be seen whether burst simulations with the new models will generate the highest observed values of WR bump/Hbeta $\approx 1$.

\subsection{SUBTYPE DISTRIBUTION}

Variation of subtype distribution with radius in the galaxy and between the Galaxy and the Magellanic Clouds was first noted by Smith (1968) and has been extensively studied since (Hidayat et.al. 1982; van der Hucht et.al. 1988). The most notable feature is that the later type WC stars are more strongly concentrated to the Galactic centre and are missing from the Magellanic Clouds. The suggestion that this is due to the different metallicity of the progenitors was made by Smith (1968) on circumstantial evidence. The reason is now elucidated by the models of Maeder (1990a, Smith and Maeder 1990). When the metallicity is low, the WR stage is achieved later in the evolution when the core is more "cooked" and has higher $\mathrm{C} / \mathrm{He}$ and $\mathrm{O} / \mathrm{C}$ ratios. (Smith and Hummer 1988) observe a strong increase of $\mathrm{C} / \mathrm{He}$ abundance to early subtypes. Smith and Maeder (1990) hypothesise that the surface composition is the primary parameter determining subtype. The models then predict (correctly) that WC9 stars are only possible when the metallicity is high; in low $\mathrm{Z}$ regions such as the LMC, only WC4 stars are possible; in even lower $\mathrm{Z}$ regions, such as the SMC, only WO stars are possible

It follows that in dwarf galaxies, with predominantly low metallicity, only WC4 and WO stars are expected. In ordinary galaxies, giant HII regions with higher $\mathrm{Z}$ should be able to produce later types; however, with only the strongest lines showing over the $O$ star continuum, WC4-6 cannot be differentiated. To produce WC8\&9 stars which are conspicuously different (see Section 2.4), requires regions with $\mathrm{Z}>\mathrm{Zo}$; such regions deserve careful observation.

The factors controlling WN subtype are not yet so clear. Identifying WNL/WNE with stars with/without hydrogen (a generalisation with many exceptions), Maeder's (1990b) models show that WNL evolve predominantly from the more massive stars. (This has been suggested often before, eg. Langer 1987, on the basis of the higher luminosity of the WNL.) However, at $\mathrm{Z}<\mathrm{Zo}$, where models predict largely WNL, the observations (LMC and SMC) show large numbers of WNE stars. The discrepancy may be due to the increasing importance of binaries. 
Thus, our uncertain expectation for dwarf galaxies is that the dominant WN subtype with be WNL. With the signal/noise available in spectra of dwarf galaxies plus the uncertainty of the line ratios, it is hard to tell the difference (see Section 2.4). As a curiosity, note that R136, the central cluster of 30 Dor and HD 97950, the central cluster of NGC 3603 have broader HeII4686 than is characteristic of WNL stars (35 A versus 20-30 A). Line width is not, however, a reliable subtype indicator.

\subsection{CALIBRATION OF LINE FLUX IN THE WRbump AT 4650.}

The estimate of $1 \mathrm{E} 36 \mathrm{ergs} / \mathrm{sec}$ for the flux from a single WR star in the 4650 feature originates from Kunth and Sargent (1981) and is based on average EW's and absolute magnitudes. AKS use $1 \mathrm{E} 36 \mathrm{erg} / \mathrm{sec}$ for a WN star and 4E36 for a WC star.

With the advent of CCD's, direct measurements of flux have become easy and a significant number of stars have been observed.

For WC stars:

* Smith et.al. (1990a, henceforth SSMa) find that, for LMC WC4-6 stars, the flux in the 4650-4686 feature is the same for all stars: $\log \mathrm{F}(\mathrm{erg} / \mathrm{sec})=36.7 \mp 0.15$ (assumes $\mathrm{M}-\mathrm{m}=18.5 \mathrm{mag}$, corresponding to $\log F / f=47.5$.) However, Smith et.al. (1990b, henceforth SSMb) find that the value for Galactic WC5-7 stars appears to be lower by about 0.5 dex.

* Line fluxes for WO star are probably lower than for WC4. For Br 93 in the LMC, the 5808 flux may be 0.0 to 0.5 dex fainter than for the WC4 stars and the 4650 flux even lower. For the WO star in the SMC, the 4650 flux is about 0.6 dex fainter than the value above (for $M-m=18.8$, EW from Conti 1989 , colours from Smith 1968a and equations from SSMa). Care is needed; the WO stars are a very diverse class (see Smith and Maeder 1990; Torres-Dodgen and Massey 1987).

For WN stars:

* Conti and Morris (preprint) find that, for LMC WN stars, the Hell4686 flux covers a wide range. Averaging over the five WN6\&7 stars in the 30 Doradus region, yields $\log F(\mathrm{erg} / \mathrm{sec})=36.5 \mp 0.6$. WNL in other parts of the LMC are few and have are not yet measured for fluxes; WNE stars in Conti and Morris' sample yield $\log F(\mathrm{erg} / \mathrm{sec})=35.970 .3$.

* Kunth and Schild (1986) specify that they include only HeII4686 in the flux measurements. AKS's WR bump measure includes NIII4640 and HeII4686. Hence the ratio of these two lines is relevant. * The ratio of NIII4640/HeII4686 is uncertain. Data of Conti and Massey (1989) and unpublished data which I hold in conjunction with Moffat and Shara indicate that NIII4640 is weaker relative to HeII4686 for LMC stars than for Galactic ones. The correction for Galactic WN7 stars is about 0.16 dex and for LMC WN7 stars is about 0.06 dex which we can neglect compared to the scatter of 0.6 dex in the flux of HeII4686.

For both sequences:

* Maeder's (1990a,b) models indicate that all WR stages will be more massive and therefore more luminous in total flux in regions of lower metallicity. Whether this will reflect directly in the line fluxes is not clear, but the difference found by SSMb indicates that it does.

Conclusion: The flux in the WRbump is probably metallicity dependent. The average values for LMC stars (assuming $M-m=18.5$ ), $\log F($ ergs/sec) $=36.5$ for HeII4686 in WN6-7 stars, and 36.7 for CIV4650 + HeII4686 in WC4 stars are probably good average values for single WR stars in regions of moderately low metallicity $\left(\mathrm{O} / \mathrm{H}^{n}=8.2\right.$ to 8.5$)$. However, for both higher and lower metallicity regions, the WC value is probably too high and the WN value is untested. For Galactic and LMC WN7 stars, the ratios of NIII4640/HeII 4686 are approximately 0.5 and 0.15 , respectively, representing a small correction on a fairly uncertain flux value for HeII4686. 

THE SPECTRA.

Two questions appear relevant:

1. What other features may be visible apart from the WRbump at $4650 \mathrm{~A}$, which may be useful as subtype indicators?

2. How can we separately estimate the numbers of WN and WC stars contributing to the WRbump?

CCD spectra of WC stars in the Galaxy and the LMC have been published by SSMa\&b. In collaboration with Shara and Moffat, I hold a collection of CCD spectra of WN stars in the Galaxy and the LMC; from these, I have extracted the generalisations below regarding strengths of emission lines in the wavelength range $4000-6000 \mathrm{~A}$.

In WN spectra, HeII4686 is the strongest feature rivalled only by NIII4640 in WN8 stars. HeII4686 > NIII4640 always. Next strongest is NV4605-20 (WN3-4) or NIV4057 (WN5-7) or NIII4640 (WN6-8), a factor 2-5 down (for Galactic stars), followed by Hell5411, a factor $\sim 5$ down compared to HeII4686. HeI 5875 is significant for WN7-8. (Relative strengths here are in peak/continuum flux which is more conspicuous and better defined in blended features.)

WC spectra have two strong features, HeII+CIII/IV4650 and CIV5808. In the 4650 region, $4650>4686$ always. In WCE spectra, these two lines are by far the strongest; in WCL spectra, CIII5696 becomes equal to CIV5808 in WC8 and exceeds it in WC9. (WC8 stars have been found in M31 but no WC9 stars have yet been seen in an external galaxy.)

These differences should allow simple discrimination between WN and WC dominance and permit estimates of how many of each sequence is present.

Figure 1 shows a combination of a WN7 spectrum (Br90 in the LMC) with a WC4 star (Br10). Br10 is twice as bright, in line and continuum, as any other WC4 star in the LMC, so I count it as two "average" WC4 stars. Notice:

* The flux scale is $-2.5 \log \mathrm{f}$ (lambda). The top two spectra are at their real magnitudes. The "double" WC4 star is about 2 mag fainter in the continuum, but the flux in the 4650 feature is about 4 times as great.

* The lower three spectra are shifted downwards to obtain good separation and represent a combination of WN7+WC4 in ratios: 1:2, 3:1, 20:1 .

* The shorter wavelength peak is always at $4640 \mathrm{~A}$; the WC feature forms a broad pedestal for the narrower features and becomes inconspicuous in the blend.

* Peak $4686 \gg>4640$ always .

* Even at 20:1 dilution, the CIV5808 is predominantly from the WC star; the WN star contributes about one third of the flux.

Clearly, separation of WC and WN star contributions using 4650 alone is not easy. However, the 5808 feature can assist.

* For WC stars, the ratio of 5808/4650 is a slowly varying function of subtype (SSMb). The flux ratio for both LMC and Galactic WC4 stars is $-0.22 \mp 0.1$ dex. (i.e. 4650 is 1.7 times stronger). (The EW ratio is $\sim+0.2$ dex, i.e. 5808 is stronger.) Thus, if WC stars are dominant, the 5808 line will be comparable to 4650; the strength of 5808 can be used directly to estimate the contribution of the WC stars to the blend at 4650 . Note that we expect (in these low metallicity galaxies) that most of the WC stars will be WC4; should they be WC6, the flux ratio is $\sim-0.3$ dex (EW ratio $\sim 0.1$ ) only 0.1 dex different from WC4\&5 stars. However, if they are WVO stars, the EW ratio may be as high as 0.8 dex (Smith and Maeder 1990). 


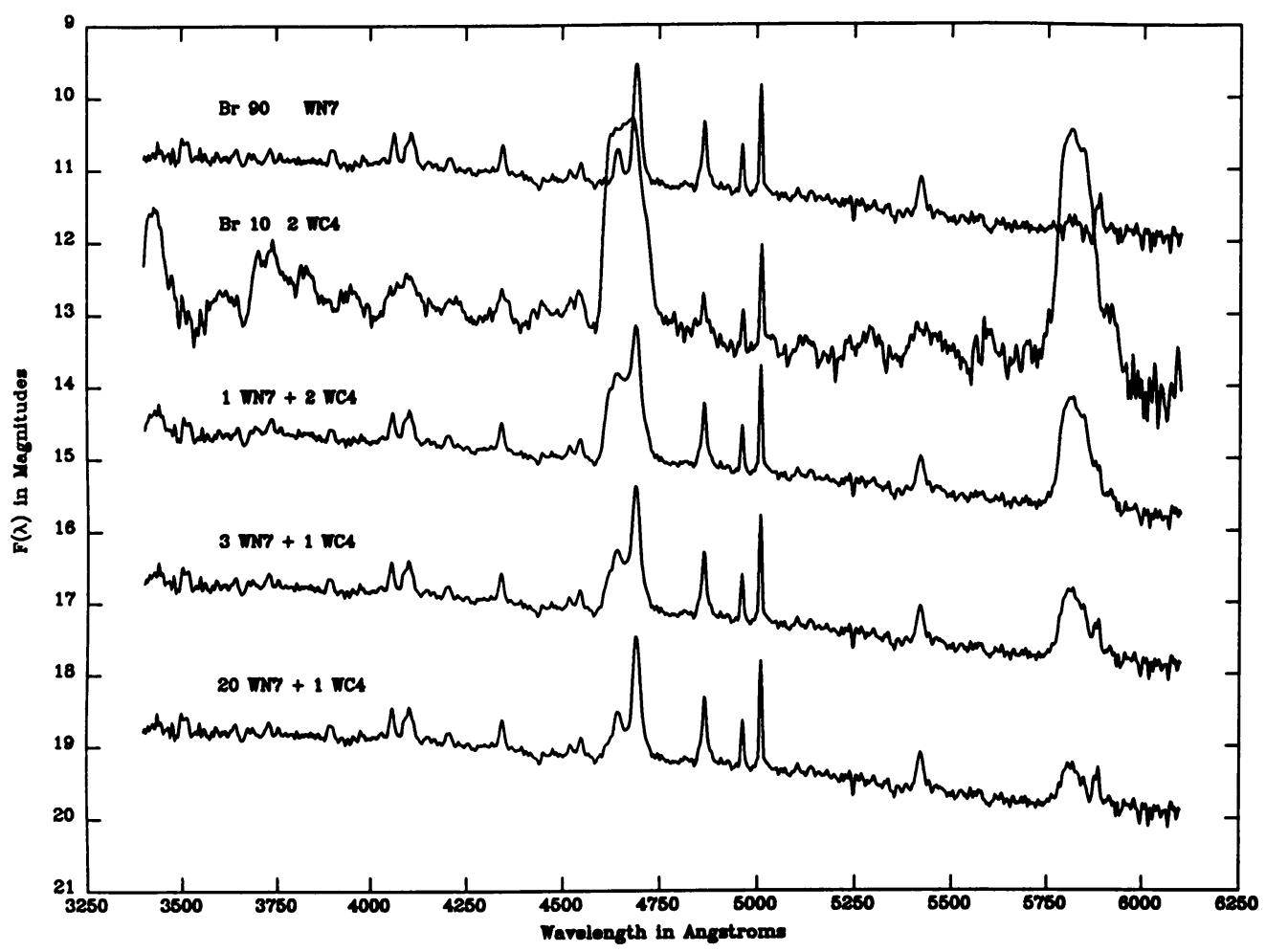

Figure 1. A combination of $\mathrm{Br} 90$ (WN7) and $\mathrm{Br} 10$ (two WC4 stars). The top two spectra are at their real magnitudes. The lower three spectra are shifted downwards to obtain good separation and represent a combination of WN7+WC4 in ratios: 1:2, 3:1, 20:1 .

TABLE 1. Average log F(erg/sec) for LMC Stars

\section{CIII/IV4650+HeII4686 CIV5808}

WC4

WN7

36.7 (a)

$36.5(\mathrm{~b})$
(a) SSMa.
(b) Mean of 30 Dor stars in the sample of Conti and Massey (1989).
(c) Smith, Shara and Moffat (in preparation). 
CIV5808 in WN stars is relatively weak but needs to be allowed for since the WN/WC ratio is usually large; the correction is uncertain because the CIV5808 strength in WN spectra is highly variable and probably $\mathrm{Z}$ dependent. Table 1 gives the mean fluxes for $\mathrm{LMC}$ stars in the two lines. On the assumption that the LMC stars are characteristic of low $\mathrm{Z}$ regions such as found in the BCD's, these values allow the quantitative separation of the WN and WC.

Comparing Figure 1 with published spectra of $\mathrm{BCD}$ galaxies, we find that most metal poor $\left(\mathrm{O} / \mathrm{H}^{\prime \prime}<8.6\right)$ BCD's appear to be pure WN, showing HeII4686 and only a hint of NIII4640 (see eg. Kunth and Sargent 1981).

WC dominated spectra have not been observed in BCD's. The average spectrum of NGC604 (d'Odorico and Rosa 1981) is a nice example from a giant HII region in a nearby galaxy.

Intermediate types are found in a few BCD's and some giant HII regions. However, these spectra all share an important difference from the ones in Figure 1: namely, the peak flux at $4640>4686$ ! This is not possible with WN6-8+WC4 of the LMC variety. In order of decreasing 4640/4686, we have: Mrk 710 (Kunth and Schild 1986), He2-10 (see below); NGC 6764 and Mrk 309 (Osterbrock and Cohen 1982); NGC 5430 (Keel 1982); NGC 5128\#13 (Mollenhoff 1981).

The case of He2-10 is ambiguous. The spectrum given by d'Odorico et.al. (1983) appears to have broad CIV4650 > Hell4686 as expected. The spectrum given by Allen et.al. (1976) (only of the blue region) shows broad NIII4640 > HeII4686. It is probable that the slit positions were different and include different WR stars (see Rosa and d'Odorico 1986, who emphasis this point strongly). What makes the thing odd, is that the shape of the feature is identical in the two observations; only the wavelength scale is different.

The clue to these unexpected spectra appears to be that, with the exception of NGC5430, they all have

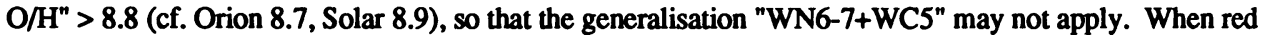
spectra are available (Mrk309, NGC5430?, NGC5128, He2-10), they all appear to have evidence of a WC contribution. If a Galactic WN7 star had been used in Figure 1, the NIII4640 line would be a factor $\sim 3$ stronger. However, to get the extreme spectra of Mrk710, a ratio of 4640/4686 $\gg 1$ appears to be needed, implying WN stars of low ionisation and/or high N/He abundance ratio.

\subsection{DIFFERENCES BETWEEN GALAXIES}

The generalisation current in the literature is that stars of the same subtype in different galaxies appear to be the same. However, evidence to the contrary is becoming convincing.

* SSMb find that the flux in the CIV5808 line is 0.5 dex lower in WC5-7 stars in the Galaxy than for WC4-6 stars in the LMC.

*SSMa find that the ratio of C/O lines is greater in the WC4 stars in the Galaxy than in the LMC.

* Smith and WIllis (1983) find that the EW's of HeII4686 are larger in the LMC stars than in the Galaxy.

* New data (as yet unpublished) indicates that HelI4686 is stronger relative to NIII4640, NIV4057, CIV5808 and HeI 5875 for WNL stars in the LMC compared to the Galaxy. This appears to be the same effect as noted above by Smith and Willis. Data of Conti and Massey (1989) indicate the same effect for HeII4686/NIII4640 although they do not comment on it. Note that if the reverse is true and the N and C lines in WN spectra are stronger in high $Z$ regions, it may help to explain $4640>4686$ in the galaxies noted above.

Conclusions: Evidence for differences in luminosity (total and line) and in line ratios for WR stars in different galaxies is becoming convincing. Care needs to be taken in calibrating these quantities. 


\section{Overall conclusions}

Interpretation of the broad emission in spectra of knots in BCD galaxies as due to the presence of WR stars appears to fit most expectations based on nearby galaxies and stellar evolution models.

* WR/OB star numbers increase with metallicity.

* WC stars are rare at the metallicity characteristic of dwarfs galaxies.

* Flux in the WRbump for LMC WC4 stars is $\log F(\mathrm{ergs} / \mathrm{sec})=36.7$ and for WN7 stars is 36.5 . These numbers may be representative for stars in regions of moderately low $\mathrm{Z}\left(\mathrm{O} / \mathrm{H}^{\mathrm{n}}=8.2\right.$ to 8.5$)$ but caution is needed at lower and higher $Z$.

* Numbers of WN and WC stars may be separated using the CIV5808 feature as well as the WRbump at 4650 if the assumption is made that the LMC stars are representative.

* At high Z, the spectra indicate too strong a line at 4640 A for simulation by LMC stars.

* Subtypes are difficult to differentiate due to low signal/noise and evident differences between galaxies.

Acknowledgement. It is a pleasure to thank Mike Potter of STScI for much of the data reduction of our unpublished spectra of WN stars and for preparing Figure 1.

\section{References.}

Abbott, D.C. (1982) Ap.J. 259, 282.

Armandroff,T.E. and Massey,P. (1985) Ap.J. 291, 685.

Allen,D.A., Wright,A.E. and Goss,W.M. (1976) M.N.R.A.S. 177, 91.

Arnault,Ph., Kunth,D. and Schild,H. (1989) A\&A 224, 73.

Conti,P.S. (1989) ApJ. 341, 113.

Conti,P.S. and Massey,P. (1981) ApJ. 249, 471.

Conti,P.S. and Massey,P. (1989) Ap.J. 337, 251.

Conti,P.S., Garmany,C.D., de Loore,C.W.H. and Vanbeveren,D. (1983) Ap.J. 274, 302.

D'Odorico,S. and Rosa,M. (1981) Ap.J. 248, 1015.

D'Odorico,S., Rosa,M. and Wampler,J.E. (1983) A\&A Suppl. 53, 97.

Gerola,H., Seiden,P.E, and Schulman,L.S. (1980) Ap.J. 242, 517.

Hazard,C. (1985) in D.Kunth, T.X. Thuan, J. Tran Thanh Van (eds.), Star Forming Dwarf Galaxies, Kim Hup Lee Printing: Singapore, $p 9$.

Hidayat,B., Supelli,K. and van der Hucht,K. (1982) in C.W.H. de Loore and A.J.Willis (eds.) IAU

Symposium 99, Wolf-Rayet Stars: Observations, Physics, Evolution, Dordrecht: Reidel, p 27.

Hutsemekers,D., and Surdej.J. (1984) A\&A 133, 209.

Kunth,D. (1985) in D.Kunth, T.X. Thuan, J. Tran Thanh Van (eds.), Star Forming Dwarf Galaxies, Kim

Hup Lee Printing: Singapore, p 183.

Keel,W.C. (1982) Publ.A.S.P.94, 765.

Kunth,D. (1989) in J.E.Beckman, B.E.J.Pagel (eds.), Evolutionary Phenomena in Galaxies, Cambridge

University Press p 22.

Kunth,D., and Sargent,W.L.W. (1981) A\&A 101, L5.

Kunth,D. and Schild,H. (1986) A\&A 169, 71.

Kunth,D. and Sargent,W.L.W. (1981) A\&A 101, L5.

Langer,N. (1987) A\&A 171, L1.

Massey,P. (1986) in C.W.H.de Loore, A.J.Willis, P.Laskarides (eds.), IAU Symposium No. 116,

Luminous Stars and Associations in Galaxies, Dordrecht: Reidel, p 215.

Massey,P. and Conti,P.S. (1983) Ap.J. 273, 576.

Maeder,A. (1981) A\&A 102, 401.

Maeder,A. (1990a) A\&A Suppl. in press. 
Maeder,A. (1990b) A\&A in press.

Maeder,A., Lequeux,J. and Azzopardi,M. (1980) A\&A 90, L17.

Melnick,J. (1985a) A\&A 153, 235.

Melnick.J. (1985b) in D.Kunth, T.X. Thuan, J. Tran Thanh Van (eds.), Star Forming Dwarf Galaxies, Kim Hup Lee Printing: Singapore, p 171.

Moffat,A.FJ., Niemela,V.S., Phillips,M.M., Chu,Y. and Seggewiss,W.M., (1987) Ap.J. 312, 612.

Mollenhoff,C. (1981) A\&A 99, 341.

Osterbrock,D.E. and Cohen,R.D. (1982) Ap.J. 261, 64.

Rosa,M. and D'Odorico,S. (1986) in C.W.H.de Loore, A.J.Willis, P.Laskarides (eds.), IAU Symposium No. 116, Luminous Stars and Associations in Galaxies, Dordrecht: Reidel, p 355.

Sargent,W.L.W., and Searle,L. (1970) Ap.J. Letters, 162, L155.

Searle,L., Sargent,W.L.W., and Bagnuolo,W.G. (1973) Ap.J. 179, 427.

Scalo,J.M. (1986) in C.W.H.de Loore, A.J.Willis, P.Laskarides (eds.), IAU Symposium No. 116, Luminous Stars and Associations in Galaxies, Dordrecht: Reidel, p 451.

Smith,L.F. (1968a) M.N.R.A.S. 140, 409.

Smith,L.F. (1968b) M.N.R.A.S. 141, 317.

Smith,L.F. (1988) ApJ. 327, 128.

Smith,L.F. and Hummer,D.G. (1988) M.N.R.A.S. 230, 511.

Smith,L.F. and Maeder,A. (1990) A\&A, in press.

Smith,L.F., Shara,M.M. and Moffat,A.F.J. (1990a) Ap.J. 348, 471.

Smith,L.F., Shara,M.M. and Moffat,A.FJ. (1990b) Ap.J. in press.

Smith,LJ. and Willis,AJJ. (1983) A\&A Suppl. 54, 229.

Talent,D.L. and Dufour,R.J. (1979) Ap.J. 233, 888.

Terlevich,R. (1985) in D.Kunth, T.X. Thuan, J. Tran Thanh Van (eds.), Star Forming Dwarf Galaxies, Kim Hup Lee Printing: Singapore, p 393.

Torres-Dodgen,A.V. and Massey,P. (1987) Ap.J.Suppl. 65, 459.

van der Hucht,K.A., Hidayat,B., Admiranto,A.G., Supelli,K.R. and Doom,C. (1988) A\&A 199, 217. Walborn,N. (1986) in C.W.H.de Loore, A.J.Willis, P.Laskarides (eds.), IAU Symposium No. 116, Luminous Stars and Associations in Galaxies, Dordrecht: Reidel, p 185. 


\section{DISCUSSION}

Moffat: When I was a young student back in 1968, I remember being strongly influenced by a colloquium you gave at the Bonner Sternwarte on WR stars. Today, I had the same sentiment! One question I still have concerns the predicted masses for WO stars at low $Z$ vs. WO masses at high $Z$. Observations of masses in WR+O binaries do not agree with the predictions, e.g., the WO4 star in the SMC binary AB8 has a mass of $5 M_{\odot}$ (cf. poster at this symposium) compared to $\sim 30 M_{\odot}$ predicted at $Z=0.002$. (I am not convinced that WR properties, including masses, in all but the very closest binaries are any different from single WR star properties).

Smith, Lindsey: I emphasize that the models are for single stars and indeed predict much higher minimum masses at low metallicity. However, the phenomenon is due to slower removal of the hydrogen envelope. If you have a binary close enough to cause mass exchange soon after the star leaves the MS, its evolution to a WC star is equally rapid at any $Z$.

Niemela: Both CIII-IV4650 and CIV5808 are seen as nebular lines in some very hot $H I I$ regions. How much would their contribution be to the WR "bumps"?

Smith, Lindsey: Nebular carbon at $4650 \AA$ is not considered by the observers. What does appear to be present is forbidden iron at $4658 \AA$. At low resolution, care is needed to not confuse this with WR carbon.

Vanbeveren: The WR binary frequency in the Galaxy is about $30-40 \%$; in the SMC it is about $20 \%$. So, it looks like the WR binary frequency increases with decreasing $Z$. Do you not think then that most of the WR stars in these dwarf galaxies are binaries and that a comparison with the evolutionary computations of single stars is inappropriate here?

Smith, Lindsey: Your point is valid. However, the agreement in subtypes predicted by single star evolution and those observed is impressive. It is hard to tell from such a great distance what is going on.

Spurzem: In discussions of the mass loss rate of WR stars is an artificial distinction between single and binary stars. Especially in dense clusters of massive stars, as they may form in starburst regions, frequent close encounters between single stars will enhance the mass loss rate due to tidal interactions.

Smith, Lindsey: It strikes me as an interesting thought, which I have not even discussed with the theoriticians. But if you have a binary, interaction may also affect the internal mixing in the stars, and would therefore also affect the subclass frequency. But I am not a theoritician, that is out of line. [See also Maeder, p. 228. (Eds.)]

Conti: I would like to comment on requiring a high binary frequency in metal-poor environments to provide WR stars. The solar vicinity has a well determined binary fraction of $40 \%$. The SMC has 5 out of $8 \mathrm{WR}$ stars known as established binaries. By binaries, I mean those with an established orbit. Thus the SMC, with low metallicity, could have $100 \%$ binaries, considering small number statistics. But what of the LMC with intermediate metallicity? Does it also have relatively many WR binaries? (Again, orbits, not just line of sight companions).

Moffat: The binary frequency among WN6/7 stars (for which the statistics are fairly reliable) is identical in the LMC and the Galaxy ( 57\%), (Moffat, 1989). The numbers are binary/total WN6/7 =5/9 in LMC, and 11/19 in the Galaxy, based on confirmed orbits or constant radial velocity. There is no obvious correlation of binary frequency with metallicity.

Underhill: $N I I I 4634-40$ as strong as or stronger than HeII4686 is the signature of Of stars. Thus, when such ratios are observed in galaxies there may be a large population of Of stars present. 
Smith, Lindsey: Yes, I noticed that in the spectra shown yesterday. The problem is that the emission line strength in Of stars is much less than in WR stars. The number of WR stars needed is already enormous; the number of Of stars would be even greater.

Walborn: The WR population of giant $H I I$ regions will be dominated by the descendants of the most massive stars, which are luminous WNL's. However, 30 Dor is embedded in a 10 times larger, less extreme young region containing numerous WC's. At great distances, the angular scales will be very small, and as Lindsey has shown, the early WC can match 20 WNL's spectroscopically. Therefore, the interpretation of stellar populations in distant starbursts is a very difficult problem.

Smith, Lindsey: It is noted in the written manuscript that the 30 Dor cluster is even younger than the predicted onset of the main WR phase. The members of stars in this region may increase dramatically in the next million years.

Maeder: Your superb presentation demonstrates us how blue compact galaxies bring a lot of very useful indications on massive star evolution at low and moderate metallicities. Regarding some comments made on binary evolution, I agree that at low $Z$ evolutionary models predict less WR stars than observed and this might suggest that at low $Z$ the rare WR stars may mainly result from binary evolution.

Shara: Under what circumstances, if any, would you think it prudent to use the constancy of flux in emission lines in WC stars as a distance indicator?

Smith, Lindsey: My guess is that the dominant parameter is the metallicity. So, if you have a region with the same metallicity as the LMC, then the LMC star values apply. If you go to higher or lower metallicity, the fluxes appear to decrease.

Mike Shara, Lindsey Smith, Tony Moffat

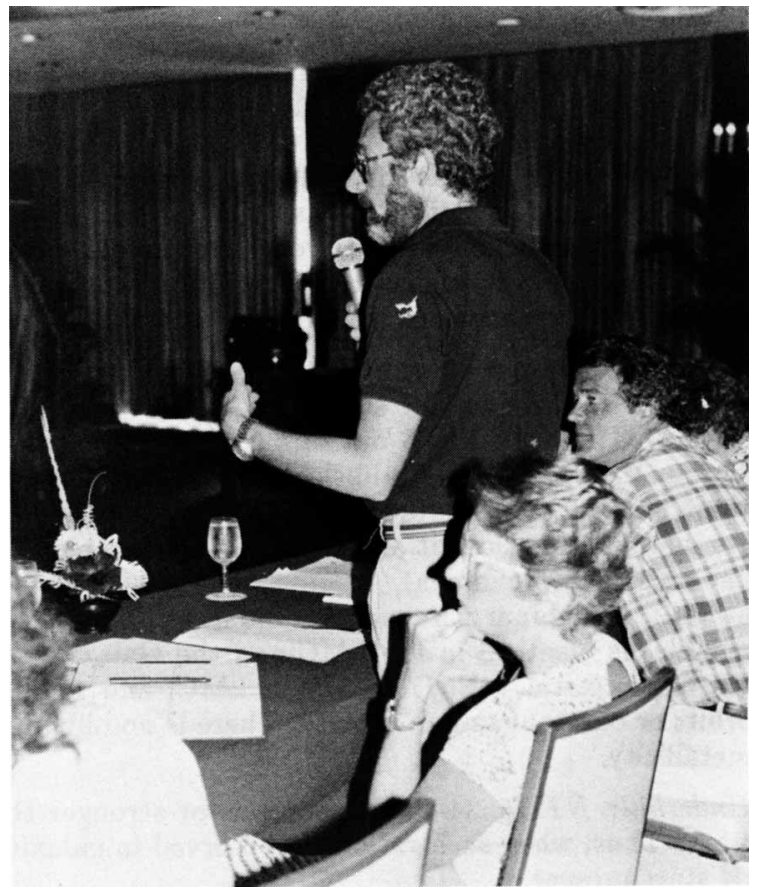

